\title{
Kalter Krieg oder innere Einheit? Der Wandel des Mediendiskurses über Ostdeutschland durch ostdeutsche Journalist*innen in Die Zeit
}

\author{
Antje Glück
}

Keywords: Marginalisierung, Journalismus, Ostdeutschland, Diskursanalyse, Transformation

\section{Abstract}

In den vergangenen drei Jahrzehnten fiel die mediale Darstellung Ostdeutschlands oft negativ aus. Ostdeutsche wurden stereotyp als rechtsradi$\mathrm{kal}$, passiv und exotisierend dargestellt, und der Osten selbst als ein $\gg$ fremdes Land« konstruiert. Dies betraf fast alle überregionalen, im Westen verankerten Nachrichtenmedien. Zeitgleich zum Aufstieg der rechtspopulistischen AfD vor allem im Osten kam es jedoch auch journalistisch zu einer Wende: Mit Zeit im Osten eröffnete das erste Redaktionsbüro, das ausschließlich mit Ostdeutschen besetzt ist. Wie wirkte sich das auf den Diskurs in der Zeit aus? Dieser Beitrag untersucht diskursanalytisch den Zeitraum von 2015 bis 2019. Als treibende Kraft tritt hier eine neue Generation von jungen selbstbewussten Journalist*innen hervor: die Wendeund Post-Wende-Kinder, geboren im Osten, aufgewachsen in Gesamtdeutschland. Der Beitrag untersucht, inwieweit ein journalistischer Wandel und integrativerer Mediendiskurs durch diese Generation realisiert wird.

Ich danke den Kollegen Armin Scholl und Michael Higgins sowie den Herausgeber*innen dieses Bandes für Kritik und Hinweise zum Beitrag. Ebenfalls danke ich meinen Eltern. 
Antje Glück: Kalter Krieg oder innere Einheit? Der Wandel des Mediendiskurses über Ostdeutschland durch ostdeutsche Journalist*innen in Die Zeit. In: Nils S. Borchers, Selma Güney, Uwe Krüger und Kerem Schamberger (Hrsg.): Transformation der Medien - Medien der Transformation. Verhandlungen des Netzwerks Kritische Kommunikationswissenschaft. Frankfurt am Main: Westend 2021. DOI: https://doi.org/10.53291/AAMU5370.

Dr. Antje Glück | Bournemouth University (UK) | AGlueck@bournemouth.ac.uk

\section{Einführung}

Mit den Feiern zum 30. Jahrestag des Beitritts der DDR zur BRD scheint die Deutsche Einheit längst vollzogen. Umso auffälliger erschien jedoch eine Reihe von Kommentaren zum Osten Deutschlands, die in den letzten fünf Jahren in politischen und medialen Diskursen auftauchten. Mal ging es um »Dunkeldeutschland«, wie es der ehemalige Bundespräsident Joachim Gauck nach einem Anschlag auf ein Flüchtlingsheim im sächsischen Heidenau formulierte, bewusst oder unbewusst anknüpfend an eine lang etablierte westdeutsche Wende-Rhetorik (Ondreka 2015). Ein andermal wurde sogar die provokante Idee eines »innerdeutschen Kolonialismus« aufgeworfen, bei der Westdeutschland eine Kolonialisierung des Ostens zugeschrieben wurde (Oberender 2017).

In diesem Beitrag argumentiere ich, dass sich zwar mit dem Beitritt der DDR zur BRD im Oktober 1990 die politisch-topographischen Grenzen der Bundesrepublik erweitert haben, dass dies jedoch nicht gleichermaßen für die räumliche Grenzziehung im Journalismus gelten muss. Die Erweiterung der politischen Grenzen deckt sich nicht unbedingt mit dem diskursiven Raum der bundesdeutschen Öffentlichkeit.

Der Nachrichtenjournalismus spielt in diesem Gefüge eine entscheidende Rolle, denn er dient nicht nur als Primärvermittler konstruierter sozialer Wirklichkeiten, sondern auch als zeitgenössisches Gedächtnis und Träger von Erinnerung. Gerade im ostdeutschen Fall spielt diese in welcher Form sie auch vorliegt - als Nachfolgeprodukt des Kalten Krieges eine unterschwellige Rolle. Über die letzten drei Jahrzehnte hinweg prägte eine distinkte Tendenz das Bild von Ostdeutschland, denn die Berichterstattung über das Territorium der ehemaligen DDR war über lange Zeit negativ geprägt.

Im Folgenden wird untersucht, ob die Berichterstattung über Ostdeutschland nach drei Jahrzehnten diesen Negativ-Bias behalten hat oder 
ob inzwischen ein Wandel zu verzeichnen ist. Offenbart sich vielleicht eine Transformation hin zu einem Journalismus, der eine Integrationsleistung zu erbringen vermag, die über Jahrzehnte defizitär war? Am Beispiel der Ost-Berichterstattung der meinungsführenden (west)deutschen Wochenzeitung Die Zeit werden Perspektiven von Transformation in journalistischen Diskursen und Praktiken aufgezeigt und bewertet.

\section{Deutsch-deutsche Erinnerungslandschaften}

Eine empirische Analyse der medialen Wahrnehmung von Ostdeutschland ist kaum möglich ohne eine tiefere Kenntnis der Struktur von gegenwärtigen deutsch-deutschen Erinnerungsdiskursen. Konkurrierende Erinnerungsentwürfe sind gegenwartsaktuell, denn sie strukturieren sowohl die Identität von Gruppen als auch soziale Realitätsentwürfe (Halbwachs 1992). Hierbei kann davon ausgegangen werden, dass in der heutigen Wahrnehmung des Ostens einerseits frühere Wahrnehmungsmuster fortwirken, sowohl aus der Zeit des Kalten Krieges als auch der Wende- und Nachwendezeit, sich diese Muster jedoch andererseits transformiert haben oder neu gebildet wurden. Sie aufzudecken ist das Ziel der vorliegenden Analyse.

Hilfreich dafür ist die Orientierung an Martin Sabrows (2009) Ausführungen zu den gegenwärtig miteinander konkurrierenden Erinnerungslandschaften an die DDR. Der Kampf darum, wie die DDR also (nicht) erinnert wird oder werden soll, spielt auch unweigerlich in die mediale Wahrnehmung des Ostens mit hinein - und dies wirkt bis in die Gegenwart fort. Sabrow unterscheidet drei primäre Gedächtnisstränge: Diktatur-, Arrangement- und Fortschrittsgedächtnis. Diese Modi Operandi des Gedenkens formen ein »tripolare[s] Kräftefeld« (ebd., 20) und greifen teilweise auf dominante Wahrnehmungsmuster des Kalten Krieges zurück.

Das Diktaturgedächtnis konzentriert sich auf die DDR als Unterdrückungs- und Unrechtsstaat. Es hält insbesondere Erinnerungen an "Schreckensorte der kommunistischen Herrschaft [...] bis zum Bespitzelungssystem der Staatssicherheit« (ebd., 18) wach und betont den Täter-Opfer-Gegensatz sowie individuelles Leid. Die DDR wird hier als negatives Kontrastbild zum Rechtsstaat präsentiert. Dieses Narrativ hat vornehmlich den staatlichen und öffentlichen Diskurs bis in die Gegenwart hinein dominiert. Vor allem in Ostdeutschland verbreitet ist hingegen das Arrangementgedächtnis, das »vom richtigen Leben im falschen 
weiß und die Mühe des Auskommens mit einer mehrheitlich vielleicht nicht gewollten, aber doch als unabänderlich anerkannten oder für selbstverständliche Normalität gehaltenen Parteiherrschaft« erinnert (ebd., 19). Diese Perspektive schaut auf die private Lebenswelt und -realität der DDR-Bürger*innen. Es betont den Stolz auf das Erreichte und Geschaffene, an glückliche Zeiten und einstige Werte. Unter diese Gedächtnisform fällt auch Ostalgie. Die dritte Form ist schließlich das Fortschrittsgedächtnis. Dieses erinnert an die DDR als idealistische legitime Alternative zu einer kapitalistischen Gesellschaftsordnung. Hierunter fallen beispielsweise Diskussionen um sozialistische Bauten und antifaschistische Gedenkorte, soziale Sicherheit und Gerechtigkeit, ein fortschrittliches Gesundheits- und Bildungssystem sowie die staatlich durchgesetzte Gleichstellung der Frau.

Ein Abgleich dieser drei Erinnerungsdiskurse mit der aktuellen überregionalen Berichterstattung macht deutlich, dass diese vor allem vom ersten Narrativ, dem Diktaturgedächtnis, geformt wird (siehe Abschnitt 4 zum Stand der Forschung weiter unten). Passende Erklärungsansätze finden sich in Ansätzen zu medialem Elitenbias und -konsens. Generell ist hier eine Nähe des Journalismus zur Politik anzunehmen. Diese äußert sich beispielsweise durch Indexing (Bennett 2011) sowie über Faktoren wie Nachrichtenselektionsroutinen und die Macht institutionalisierter Quellen (vgl. Krüger in diesem Band). Eine weitere Rolle spielt die Objektivitätsnorm, der zufolge Ansichten, die keine politische Rückendeckung haben, als Bias interpretiert werden können. Hinzu kommt, dass politische Eliten in der Lage sind, Vergangenheiten selektiv zu thematisieren und somit zu manipulieren, um die eigene Macht zu sichern (Hobsbawm und Ranger 1983).

Es kann jedoch nicht davon ausgegangen werden, dass Viktimisierung durch den Staat eine universale oder Mehrheitserfahrung in der DDR war (Hogwood 2013). Aus diesem Grund lässt eine journalistische Berichterstattung, die primär auf das Diktaturgedächtnis ausgerichtet ist, andere Wahrnehmungen und Erinnerungen an die DDR und die Nachwendezeit außen vor. (Westdeutsche) Elitendiskurse und heterogene ostdeutsche Erinnerungsnarrative weichen stark voneinander ab. ${ }^{1}$ Damit wird die Heterogenität und Multidirektionalität ostdeutscher

1 Jedoch gibt es auch innerhalb der westdeutschen Diskurslandschaft gravierende Unterschiede, so zum Beispiel der Mythos der Befreiung des Ostens durch Westdeutschland versus die Kolonialisierung des Ostens durch die ideologisch anders ausgerichtete BRD (Hogwood 2013, 41). 
Erinnerungskonstruktionen unzureichend abgebildet. Dies kann problematische Auswirkungen auf die Identität von Ostdeutschen haben, da soziale Gruppen Erinnerung über die Definition von Vergangenheit bilden (Halbwachs 1992).

Letztendlich ist es schwierig zu definieren, was eigentlich 30 Jahre nach dem Ende der beiden deutschen Staaten genau ost- und was westdeutsch ist. Beide Identitäten sind geschichtlich jung und gründeten sich auf die Nachkriegszeit nach dem Zusammenbruch Hitlerdeutschlands. Hier wurden je nach politischer Blockzugehörigkeit im Kalten Krieg zwei unterschiedliche Identitäten geformt, die miteinander in direkter Konkurrenz standen. Das Zusammenspiel der Betonung negativer Erinnerungen an die DDR durch die bundesdeutsche Politik soll zusammen mit der Abwertung positiver Erinnerungen idealerweise zu einer gesamtdeutschen Identität führen, die auf rein westdeutschen soziopolitischen Normen beruht (Hogwood 2013, 42). Dies bildet den Rahmen für eine asymmetrische Machtkonstellation.

Der nachfolgende Abschnitt entwickelt genauer, wie aktueller Meinungsjournalismus und gesellschaftliche Erinnerung zusammenhängen.

\section{Journalismus als Träger gesellschaftlicher Erinnerung}

Journalismus verstanden als »culturally constructed narratives« (Bird und Dardenne 1988, 67) besitzt die Wirkmächtigkeit, durch die Herstellung von Öffentlichkeit gesellschaftliche Diskurse zu definieren und damit überindividuell gültige soziale Wirklichkeiten zu schaffen. Der Informationsjournalismus erfüllt hier eine wichtige Funktion: Er ermöglicht potenziell Teilhabe und Mitbestimmung aller gesellschaftlichen Akteur*innen, indem er »Orientierung in einer komplexen Welt« bietet (Meier 2018, 14). Diesem Potenzial steht jedoch entgegen, dass Journalismus am vollständigen Beschreiben der Realität nur scheitern kann - jedoch liegt seine Stärke im Erzählen von Geschichten über die Realität (Köhler 2009).

In diesem Sinne lassen sich für den Gegenwartsjournalismus zwei grundlegende Charakteristiken identifizieren: Zum einen erscheint Berichterstattung allgemein als institutionalisierte Erzählerin von Geschichten auf gesamtgesellschaftlicher Ebene, zum anderen erlaubt ein interdisziplinärer Rückgriff auf Memory Studies das Verständnis von 
Journalismus als kollektiver Erinnerungsträger von Vergangenheit, als Geschichtserzähler oder Chronist.

Journalismus als Institution gesellschaftlichen Geschichtenerzählens vermittelt Wirklichkeiten, darunter »chronicles« als Darstellungen von Ereignissen und »stories« als Einordnung und Kontextualisierung des Zeitgeschehens (Bird und Dardenne 1988). Journalistische Medien können in diesem Sinn als Plattformen sozialer Praxis gedacht werden. Sie bieten Referenzrahmen an, die strukturieren und bestimmen, ob ein Thema als relevant und ein Framing oder ein Narrativ als gültig anzunehmen ist.

Narrative umfassen hier verbale wie visuelle, rationale wie emotionale Anteile; sie sind im Sinne von Langers (1965) "präsentativer Symbolik« erfassbar als Ganzheit einer Situationserfahrung und sinnliche Unmittelbarkeit. Gruppen können dabei einen konjunktiven Erfahrungsraum teilen (Mannheim 1980, 220), verbunden durch ein geteiltes Schicksal und Gemeinsamkeiten im biographischen Erleben. Dies bestärkt eine kollektive Dimension der Wissensgenerierung. Dabei ist es wichtig hervorzuheben, dass es nicht allein um den Austausch von bereits gemachten (kollektiven) Erfahrungen geht, sondern sich auch über zukunftsbezogene Erwartungshaltungen verständigt wird. Zusammengefasst geht es beim Gegenstand dieses Beitrags also um einen konjunktiven deutsch-deutschen Erfahrungs- und Erwartungszusammenhang.

Nachrichtenmedien haben eine zentrale Stellung in diesem Verständigungsprozess inne, denn, wie Tenenboim-Weinblatt (2011) ausführt, gilt auch Journalismus als Träger von »prospective memory«. Unter diesem Konzept kann die Herleitung der Zukunft aus der Vergangenheit verstanden werden, quasi als »vorausschauende Erinnerung«. Eine solche Konzeptualisierung von Medien als zentrale Instanzen gesellschaftlicher Erinnerungen basiert auf zwei Annahmen. Erstens gibt es kein Zeitalter ohne Erinnerungskonstruktion - Vergangenheiten werden in jeder Gegenwart konstruiert. Sie bilden das kommunikative Gedächtnis einer Gesellschaft (Belke 2009, 175). Zweitens sind retroaktive (vergangene) und prospektive Erinnerung eng miteinander verbunden. Mediale Diskurse geben Vergangenheit nicht einfach in der Gegenwart wieder, sondern nutzen sie als Erfahrung für die Zukunft: »In this way, memory can be perceived as mediating between historical experience and contemporary behaviours." (Hogwood 2013, 36) Die medialen Diskurse über den Osten definieren das im vereinigten Deutschland gültige Wissen über Ostdeutschland und die Ostdeutschen. 


\section{Forschungsstand: Das Medienbild Ostdeutschlands}

Die medienwissenschaftliche Forschung hat sich intensiv mit medialem Strukturwandel in Transformations- und Zerfallsprozessen beschäftigt. Jedoch fanden Prozesse, in denen ein Staat einen anderen komplett eingliedert oder übernimmt, wenig Beachtung.

Analysiert man die Hintergründe der Medienberichterstattung über Ostdeutschland in den letzten Jahrzehnten, fallen zunächst zwei strukturelle Ungleichheiten im bundesdeutschen Mediensystem ins Auge. Westdeutsche Medien behielten auch nach dem Mauerfall die Autorität, das Bild des Ostens, eingeschlossen dessen realsozialistischer DDR-Vergangenheit, zeichnen zu dürfen. Dass diese Macht lange unangetastet blieb, liegt zuvorderst in der Etablierung spezifischer bundesdeutscher Medieneigentumsstrukturen begründet. Fast alle ostdeutschen Medien wurden bereits kurz nach der Wende in westdeutsche Hände (sprich: Verlagskonzerne) übergeben, wodurch die Entwicklung eines eigenständigen ostdeutschen Medienmarktes weitgehend ausblieb (Tröger 2019). Somit fehlten dezidiert ostdeutsche Stimmen innerhalb der bundesdeutschen überregionalen Öffentlichkeit von Anfang an. ${ }^{2}$ Aufgrund der homogenen (westdeutschen) Eigentümerstruktur sahen sich viele eingesessene bundesdeutsche Massenmedien auch in den Folgejahrzehnten kaum einer ernstzunehmenden ostdeutschen Konkurrenz - sprich: einem alternativen oder gegenläufigen Diskurs beziehungsweise Öffentlichkeit - ausgesetzt. Parallel dazu wurden mediale Elitepositionen selbst in Medien, die ostdeutsche Leser*innen- und Rundfunklandschaften bedienen, mit westdeutschem Führungspersonal besetzt (vgl. Mükke 2021).

Vor diesem Hintergrund erscheint eine marginalisierende Darstellung des Ostens aus einer dezidiert westdeutsch geprägten Perspektive als logische Konsequenz. Selbige folgt neben den Kriterien von Marktgängigkeit und Zielgruppenorientierung ebenso einer politischgesellschaftlichen Agenda. Der Osten dient der Funktion der Selbstversicherung - als Alter ist er gleichzeitig Vergleichs- und Kontrastfolie des bundesdeutschen Selbstverständnisses. Dadurch dient der in den

2 Ausnahmen bilden hier die ehemaligen DDR-Zeitungen Junge Welt und Neues Deutschland, die als Zeitungen mit regionaler Wirkung vor allem im Osten weiterbestanden, sowie der Lokalfernsehmarkt bis Mitte der 1990er Jahre (siehe dazu Kretzschmar und Steinmetz in diesem Band). 
Medien oft porträtierte Alteritätsdiskurs zur Re-Etablierung westdeutscher Identität (Ahbe 2009, Tenenboim-Weinblatt 2011).

Die kommunikations- und medienwissenschaftliche Forschung seit den 1990er Jahren arbeitet recht übereinstimmend ein eher negatives Bild der bundesdeutschen Nachrichtenberichterstattung über den Osten heraus. Es sind wenige, aber aussagekräftige Studien, die von Zeit zu Zeit empirische Bestandsaufnahmen wiedergeben. Dazu zählen Früh et al. (1999), Abhe (2009), Kolmer (2009), Früh et al. (2011), Kollmorgen et al. (2011) und zuletzt Ruhrmann (2016) oder der Mitteldeutsche Rundfunk (2018). Grob verallgemeinernd lässt sich sagen, dass diese Studien drei wiederkehrende Trends identifizieren: erstens die Unterrepräsentation und Marginalisierung Ostdeutschlands in der Nachrichtenberichterstattung, zweitens die Abwesenheit ostspezifischer Themen und drittens einen Sprach- und Bildergebrauch, der »regionsbasiertes Othering (Matejskova 2013, 35) als stets präsentes Framing des Ostens betont.

Zentral dabei ist das Grundverständnis über die Kontinuität der westdeutsch geprägten Berichterstattung. Abhe $(2009,59)$ zeigt auf, wie seit dem Wendejahr 1989/90 kontinuierlich »Wahrheiten< über die Ostdeutschen, ihre Vergangenheit und ihre Sitten in Umlauf gesetzt« wurden, welche die Basis dafür schufen, »Stereotype und Deutungsmuster zu gültigem Wissen « werden zu lassen. Die Tendenz, die in bundesdeutschen Leitmedien wie beispielsweise der Süddeutschen Zeitung oder der FAZ zu finden ist, habe »wenig mit dem zu tun, was sich die Zeitzeugen heute über die Vergangenheit erzählen - vor allem, wenn sie damals im Osten Deutschlands gelebt haben« (Meyen 2013, 225). Das gleichzeitige Fehlen authentischer Eindrücke aus den fünf neuen Bundesländern im öffentlichen Diskurs trug dazu bei, die Geschichte des Ostens und der DDR im breiten Maße abzuwerten, unter anderem durch Gleichsetzung mit dem Nationalsozialismus als »totalitäre Staaten « (Wippermann 2009, 8). Pauschale Stigmatisierungen wie diese ließen neue spezifisch ostdeutsche politische Entwicklungen (wie zum Beispiel die Partei Die Linke) eher außen vor oder betteten diese in bestehende Diskurse ein.

Besonders problematisch erscheint die unterschwellige Tendenz, Ost-West-Differenzen zu hierarchisieren. Ostdeutsche werden dabei routinehaft als Kontrast zur westdeutschen Norm gesehen, als eine schwer verständliche Minderheit eines »fremden Territoriums « (Wedl 2009), die es kaum in die Alltagswelt des Westens schaffe. Diese »hierarchisch konstruierte« Differenz schreibt dem Westen ein deutsches 
»genealogisches Erbe« zu, während ehemalige Ostdeutsche als geringwertiger eingestuft werden (Hörschelmann 2001, 986). Die Erwartungshaltung an die "Neuen « aus dem Osten ist hier Nachahmung und Anpassung an die »proper citizens « des Westens (Matejskova 2013, 34-35). ${ }^{3}$ Der Westen fungiert dabei als »Normal Null« (Roth 2008), und der Osten bildet die Abweichung und Differenz dazu (Ahbe 2009).

In der Folge müssen Ostdeutsche mit einem permanenten medialen Fremdblick leben. Damit einher geht auch die Homogenisierung der sehr heterogenen Gruppe der Ostdeutschen. ${ }^{4}$ Wedl (2009) zeigte mit einer Analyse von Zeit-Artikeln auf, wie dies diskursiv umgesetzt wird: Zum einen werden Ostdeutsche entindividualisiert als Masse dargestellt; zum anderen fungiert »ostdeutsch « weiterhin als essentialistische Eigenschaft und funktionales Alteritätskonstrukt westdeutscher Identität.

Die Abwesenheit von ostdeutschen Stimmen im Mediendiskurs ist dann besonders auffällig, wenn westdeutsche Expertise den Osten zu erklären versucht: Es wird über anstatt mit Ostdeutschen gesprochen. Jedoch zeigt sie sich dabei wenig erfolgreich, wenn es um die Popularität rechtsradikaler Bewegungen in Ostdeutschland geht. Pauschale öffentliche Verurteilungen wie die »Dunkeldeutschland«-Bemerkung des damaligen Bundespräsidenten Joachim Gauck (Ondreka 2015) gießen zusätzlich Öl ins Feuer.

Diese jahrzehntelange, allgegenwärtige diskursive Marginalisierung hat Auswirkungen auf ostdeutsche Identitätskonstruktionen und die Selbstverortung innerhalb einer gesamtdeutschen Medienöffentlichkeit. Jüngst wurde dem Westen »kultureller Kolonialismus« vorgeworfen (Krüger in Decker 2017) und das Konzept getestet, Ostdeutsche als Migrant*innen zu betrachten (Ergin 2018; Foroutan et al. 2019; Köpping 2018). Ebenso meldeten sich ostdeutsche Prominente öffent-

3 An dieser Stelle ist Kimmels $(2009,57)$ Hinweis auf die Entstehung eines unterschwelligen westdeutschen Überlegenheitsgefühls interessant. Er verweist auf den Stolz von Westdeutschen, sich nach der Aufteilung des Deutschen Reiches unter den »besseren « Besatzer*innen zu wähnen, später »das Gefühl, als `bessere ¿ Deutsche im `besseren« Deutschland zu leben«. Dieses subjektive Empfinden wurde durch die Massenfluchtbewegungen aus Ostdeutschland 1989 re-aktualisiert.

4 Die Sondersituation von Ostdeutschen als äußerst heterogene »Gruppe« macht sogar explizite Antidiskriminierungsbemühungen rechtlich kompliziert (Liebscher 2019). 
lich zu Wort mit Aufrufen wie »Integriert doch erst mal uns!« (Köpping 2018) oder »Hört endlich zu! « (Richter 2018).

Selbst die Bilanz der Bundesregierung zu 30 Jahren deutscher Einheit erwähnt, dass sich die Mehrheit der Ostdeutschen als »Bürger zweiter Klasse« wahrnimmt (Presse- und Informationsamt der Bundesregierung 2020, 14; Foroutan et al. 2019) - eine Selbstwahrnehmung, die sich seit 20 Jahren wenig geändert hat (Neues Deutschland 2001).

Interessant erscheint hier die zugespitzte Formulierung Ahbes, dass »Ostdeutsche latent als eine Spezies konstruiert [werden], die politisch als demokratieunfähig, autoritätsgläubig und ausländerfeindlich gilt, kulturell als provinziell, spießig-piefig und unselbständig « (Ahbe 2001, 793). Diese und andere empirische Ergebnisse liegen jedoch zwei Jahrzehnte zurück.

Die dargestellten Momentaufnahmen über die diskursive Konstruktion des Ostens nehme ich zum Anlass, eine kursorische Bestandsaufnahme der medialen Gegenwartsdiskurse zu unternehmen - drei Jahrzehnte nach dem Mauerfall. Anlass hierfür sind veränderte politische Gegebenheiten, darunter ab 2015 die Proteste gegen die Pro-Immigrationspolitik der Bundeskanzlerin Angela Merkel sowie die deutlichen Wahlerfolge der rechtspopulistischen AfD. Diese Ereignisse führten zu einer sehr negativ geprägten Berichterstattung über den Osten, ohne jedoch notwendigerweise Erklärungen für den »fremden Osten« zu liefern.

Zentral bleiben die von Abhe (2009) aufgeworfenen Fragen an den gesamtdeutschen Mediendiskurs: Wer prägt gültige Vorstellungen über eine Bevölkerungsgruppe? Welche Rolle spielen meinungsführende Medien? Und wie interagieren tradierte Identitäten und neue Konfliktlinien mit den bereits aufgezeigten etablierten Diskursmustern? Schließlich: Braucht Journalismus einen anderen Ansatz und normativen Begründungszusammenhang?

\section{Kritische Diskursanalyse als Annäherung an Medienbilder}

Als Material für dieses Projekt wurde das Print- und Onlineangebot der überregionalen Wochenzeitung Die Zeit ausgewählt. Das Medium gilt als meinungsführend und bietet durch die nicht-tagesgebundene Berichterstattung tiefere Reflexionsmomente über den Status quo. Von Interesse für die vorliegende Untersuchung sind vor allem die subjekti- 
ven Darstellungsformen Leitartikel, Kommentar, Feuilleton oder Reportage. Die Diskursanalyse soll einen Einblick geben, wie der Osten Deutschlands innerhalb eines relevanten gesamtdeutschen Medienbildes verankert ist.

Zur Erstellung des Textkorpus wurde in die Datenbank LexisNexis eine Suchsyntax ${ }^{5}$ eingegeben. Diese filterte alle Artikel mit expliziter Erwähnung der Suchwörter »Osten« oder »Ostdeutschland« heraus. Alle Artikel mit Syntaxbezug vom 1. Januar 2015 bis 7. Mai 2019 (Ende der Datenerhebung) gingen in die Auswahl ein. Anschließend wurde eine manuelle Feinauswahl vorgenommen, denn von Interesse waren nicht punktuelle Ereignisse oder Themen, sondern der breitere Blick auf den Osten, die Darstellung von dessen Lebensrealität und Geschichte sowie Diskussionen um ostdeutsche Identität. Ein Artikel wurde dann ausgewählt, wenn sein thematischer Schwerpunkt auf Ostdeutschland lag. Nach Anwendung dieser Kriterien und Entfernung von Dopplungen verblieben $N=296$ Beiträge. $^{6}$

Dass hier der Fokus auf der Zeit liegt, hat einen weiteren Grund: Das Büro von Zeit im Osten war (und ist) wegweisend für die gesamtdeutsche Zeitungslandschaft. In Dresden 2009 gegründet, etablierte sich die Redaktion ab 2015 in Leipzig. Unter der Leitung von ehemals Martin Machowecz und seit März 2021 Anne Hähnig bearbeitet heute eine recht junge Redaktion ostdeutsche Themen aller Art. Diese bedient die nationale Ausgabe der Zeit inklusive aller Supplements sowie die Regionalausgabe Zeit im Osten. Letztere ist zwar vollständig online zugänglich, gedruckt jedoch nur in der Regionalausgabe für Ostdeutschland. Dem Leipziger Zeit-Büro folgten Spiegel und Süddeutsche Zeitung mit kleineren »Ost«-Besetzungen ebenfalls nach - diese produzieren jedoch keine eigenständigen Ost-Ausgaben.

Zugang zur Analyse des Materials bietet die Kritische Diskursanalyse. Dieses Verfahren legt Wert auf das Sichtbarmachen unterliegender Ideologien und Machtverhältnisse oder in den Worten von Machin und Mayr: "Since language can (re)produce social life, what kind of world is being created by texts and what kinds of inequalities and interests might this seek to perpetuate, generate or legitimate?« $(2012,24)$ Der

5 Die verwendete Suchsyntax für Die Zeit lautete: »(ostdeutschland or ostdeutsch or ostdeutscher or ostdeutsche or ostdeutschem or ostdeutschen) and publication(Die Zeit)《

6 Eine weitere Quelle ist der Sonderband der Zeit 》Guter Osten, böser Osten« (Schwarz 2019), der eine Auswahl der in der Zeit veröffentlichten Beiträge über das Ost-West-Verhältnis vereint. 
Sprachgebrauch in Diskursen erscheint dabei weder als neutral noch als apolitisch, sondern als Ausdruck einer Menge von Überzeugungen (Fairclough 1989).

Diese Analyse soll primär Fragen nach den Textinhalten und möglichen Diskursveränderungen im Laufe des Untersuchungszeitraums unter zeit- und kontextsensitiven Gesichtspunkten beantworten: Welche Themen und Argumente werden angesprochen, welche nicht? Was bleibt ungesagt? Verändern sich Argumente und wenn ja, warum (»critical discourse moments«; siehe Carvalho 2008)? Diskurstransformationen werden an ausgewählten Texten illustriert. Ein weiteres Interesse liegt auf der Autor*innenschaft (Ost gegenüber West). Die Analyse prüft außerdem, inwieweit empirisch belegte Diskursmuster (siehe oben) aus den vergangenen Jahrzehnten weiterhin aktuell sind.

\section{Analysiert: Der »0sten« im Zeit-Mediendiskurs}

Der folgende Überblick beleuchtet mittels Themen- und Diskursanalyse, wie Ostdeutschland in der Zeit dargestellt wird. Sofort ins Auge fällt die ungleiche Verteilung der 296 Beiträge über den Untersuchungszeitraum hinweg. Für die Jahre 2015 und 2016 wurden insgesamt 45 Beiträge ermittelt. Allein im Jahr 2018 waren es bereits mehr als 100. Das erste Jahresdrittel 2019 enthielt bereits 66 Beiträge (siehe Tab. 1).

Tab. 1: Häufigkeiten der Beiträge in der Zeit zum Thema »ostdeutsch/ Ostdeutschland« Januar 2015 bis Mai 2019 (bereinigte Auswahl).

\begin{tabular}{|c|c|c|c|c|c|c|}
\hline $\begin{array}{l}\text { "ostdeutsch* }{ }^{*}- \\
\text { pro Jahr }\end{array}$ & Gesamt & 2015 & 2016 & 2017 & 2018 & $\begin{array}{c}2019 \\
\text { (Jan. bis Mai) }\end{array}$ \\
\hline Die Zeit & 296 & 26 & 19 & 76 & 109 & 66 \\
\hline
\end{tabular}

Während frühere medienwissenschaftliche Studien regelmäßig die Unterrepräsentation Ostdeutschlands im Diskurs nachgewiesen hatten, ist das bei der Zeit nur bis 2016 der Fall. Ab 2017 nehmen die Beiträge rapide zu. Eine mögliche Ursache dafür bilden parallele politische Entwicklungen: Die Pegida-Proteste und der Wahlerfolg der rechtspopulis- 
tischen AfD bei den Bundestagswahlen im September 2017 lenkten die Aufmerksamkeit auf den Osten. ${ }^{7}$

\subsection{Kontext: Das Leipziger Büro Zeit im Osten und Autor*innenbiographien}

Das Jahr 2017 steht auch für eine zweite journalistische Entwicklung. Zu diesem Zeitpunkt war das Zeit-im-Osten-Redaktionsbüro in Leipzig bereits fest etabliert. Innerhalb weniger Jahre entwickelte sich diese Redaktion zu einer festen Stütze des Hamburger Hauptbüros mit einer enormen Bandbreite an Themen und einer großen Anzahl von Beiträgen aus dem Osten. Beim zehnjährigen Jubiläum am 5. September 2019 in Leipzig war die Rede von »einem anderen Blick auf Ostdeutschland«. Den Redakteur*innen geht es vor allem darum, den »Osten anders zu erzählen« und zu fragen, wie dieser »zu einem selbstverständlichen Stück dieser Republik werden « kann (Freunde der Zeit 2019). Diese andere Stimme soll integrativ sein und bildet die Leitvision für den Journalismus aus, im und für den Osten - Journalismus mit einer gewissen Mission also.

Das Leipziger Büro stellt bis heute eine Anomalie in der gesamtdeutschen Presselandschaft überregionaler Zeitungen dar. Denn eine Vielzahl seiner festen und freien Autor*innen sind im Osten geboren meist kurz vor oder nach der Wende. Dazu zählt der Redaktionskern um Martin Machowecz (bis März 2021 Büroleiter von Zeit im Osten, *1988 in Meißen), Anne Hähnig (*1988 in Freiberg), Valerie Schönian (jetzt freie Autorin, *1990 in Gardelegen), aber auch weitere Autor*innen wie Caterina Lobenstein (*1983 in Jena) oder Carolin Würfel (*1986 in Leipzig). Diese letzte Generation der DDR-Geborenen kennt das frühere Gesellschaftssystem aus eigener Erfahrung kaum oder gar nicht, sondern lediglich durch die Erzählungen der eigenen Familie (neben der Schule und den Medien). Sie gelten als Wendekinder- und Post-Wende-Generation mit ostdeutscher Identität, aber unterbrochener oder keiner Sozialisation in der DDR (Ahbe und Gries 2006; Kubiak und Weinel 2016). Weitere Autor*innen erlebten als »Wendejugend « Kindheit und Jugend in der DDR (darunter Jana Simon, Jana Hensel, Christian Bangel, Evelyn Finger und Maike Nedo) und haben dadurch

7 Die AfD erreichte in Sachsen überraschend die größte Stimmenanzahl, während sie in allen anderen vier ostdeutschen Bundesländern auf dem zweiten Platz lag. 
einen realen Bezug zur DDR-Geschichte des Ostens. Nur ein einziger ostdeutscher Zeit-Autor im Sample - Christoph Dieckmann (*1956) blickt auf eine geteilte persönliche Biographie in beiden deutschen Staaten zurück.

Diese Redaktionszusammenstellung erlaubt zwei Schlussfolgerungen über Merkmale, die den Mediendiskurs über den Osten prägen. Zum einen sticht die Abwesenheit einer potenziell »belastenden« historischen Vergangenheit unter den meisten Ost-Autor*innen ins Auge, da es kaum Interaktionen mit dem Staatsapparat gegeben hat. Damit beschränkt sich reflexive DDR-Erfahrung auf Sekundärerzählungen des sozialen Umfelds, während die eigene erwachsene Identität in einem gesamtdeutschen Zusammenhang erlebt und gestaltet wurde. Zum anderen geht es um die Suche nach einer klar definierten eigenen Identität innerhalb des deutsch-deutschen Gesamtgefüges. Hier fallen durch die Abwertung der DDR ältere Ost-Generationen als Rollenvorbilder weitgehend aus und ein ausschließlich westdeutsch ausgerichteter Diskurs erscheint unzureichend. Die eigenständige Identität des Ostens rückt in den Blickpunkt (siehe Göring-Eckardt 2018 und die Initiative »Wir sind der Osten $\ll^{8}$ ). Dies kommt auch im Journalismus der Zeit deutlich zum Ausdruck - Biographie als Story - und wird nachfolgend im Abschnitt 6.6 diskutiert.

Die nächsten Abschnitte widmen sich inhaltlichen Aspekten von besonderen Diskurskonstellationen um Ost-West mit folgenden fünf Bereichen: Negativ-Stereotypisierungen, Ost und West als unterschiedlich, aber gleichberechtigt, Positivnachrichten und Emanzipation des Ostens, Thematisierung von Ungerechtigkeiten und ostdeutsche Identität.

\subsection{Diskursformation 1: Negative Ost-Stereotype}

Unreflektierte Negativstereotype, wie sie in früheren empirischen Studien aufgezeigt wurden, finden sich indirekt wieder - vor allem in Beiträgen zum Rechtsextremismus, zur DDR-Vergangenheit und zum Osten als fremdem Land, das es zu entdecken gilt.

Die überregionale Vorliebe der Themenkombination »Ostdeutschland, Rechtsextremismus und AfD « ist auffällig (20 von 24 Beiträgen). Adressiert werden Fremdenfeindlichkeit in Bautzen, Chemnitz, Dresden oder Tröglitz. Während 2015 noch ein westdeutscher Experte

8 https://wirsindderosten.de/ 
(Soziologe Heinz Bude, »Fremdenfeindlichkeit: 'Existenzieller Neid««, 05.02.2015) Pegida im Osten zu erklären versucht, finden sich hier parallel bereits Perspektiven von innerhalb der ostdeutschen Gesellschaft - so beispielsweise der Artikel von Machowecz über »Busen, Bier und Islamismus « (23.04.2015), in dem er das politische Aktivwerden von Pegida-Gründer Lutz Bachmann und seines Freundeskreises abseits von Klischees darstellt. Bei Carolin Würfel (»Ein schreckliches Heim-Weh«, 27.01.2017) vermischt sich die politische Analyse mit privaten Erfahrungen bei der Rückkehr in den persönlichen Bekanntenkreis nach Leipzig. Doch es sind vor allem die rassistischen Vorfälle in Chemnitz, über die vorrangig ostdeutsche Reporter*innen berichten. Zwar beschreibt der westdeutsche Autor Gunther Schnabl den Osten als »Leeres Land«, das die Toleranz aufgrund von Abwanderung und Armut »längst verloren« hätte (27.09.2018), doch bilden anderswo ostdeutsche Autor*innenteams eine differenzierte Alltagsrealität ab. Kreativer Widerstand gegen Rechtsextremismus wird genauso beleuchtet (»Wie klingt linke Heimatmusik? «, 11.01.2018; »Die Mitte muss brummen«, 10.09.2018) wie die Emotionen von Ostdeutschen oder der Zusammenhang von Rechtsextremismus mit Geschlecht (»Die Wut der Frauen«, 09.03.2017) oder Unternehmern (»AfD: Chef sucht ein Zuhause«, 29.01.2015). Ein Negativbeispiel ist hier allerdings Evelyn Fingers polemischer Beitrag »Stolz der Verlierer « (14.09.2017), der Ostdeutsche gleich zu Beginn dreifach auf primitive Emotionen reduziert: »Sie brüllen ihren Hass gegen die Politik hinaus«; »als die Ostdeutschen brüllten, wir seien ein Volk«; »das Gebrüll der Ossis«.

Als zweite große Dimension spielt die DDR-Vergangenheit des Ostens eine Rolle. Diese scheint oft in negativem oder bestenfalls ambivalentem Licht. So wertet man an sich positive Akte der DDR negativ um (Gedenken an die Bombardierung Dresdens: „Bomben aufs Abendland «, 13.02.2015; Internationale Solidarität: »Sie hassen die Moral der Eliten«, 25.02.2018). In »Jugendarrest: SSie fühlen sich abgehängt« (25.05.2016) beschreibt die Autorin, wie strafgefährdete Jugendliche durch Konfrontation mit einer repressiven DDR-Vergangenheit für positive Aspekte der Demokratie gewonnen werden sollen. Einige Beiträge fügen sich in das oben erklärte »Diktaturgedächtnis« ein. Jedoch gibt es auch Gegenbeispiele, die eine positiv Lebensrealität in der DDR vermitteln. Dazu zählen beispielsweise die Ermahnung zur Würdigung der Lebensleistungen Ostdeutscher (»Helden der Arbeit«, 19.09.2018) oder erfolgreiche Biographien vor und nach der Wende (Karl Döring rettet einen Betrieb in Eisenhüttenstadt über die Wende: »Bei uns war nichts 
marode«, 08.10.2015). Beide Beiträge zeigen Normalität in der DDR und bedienen damit eher Diskurse des Arrangementgedächtnisses.

Die dritte Dimension ist die partielle Exotisierung des Ostens als (weiterhin) »fremdes Land «, das durch einen Nicht-Ostdeutschen »entdeckt « wird. Dieses fortbestehende Motiv fand sich im Untersuchungszeitraum mehrfach. So besuchte Moritz von Uslar sowohl Görlitz als auch den Weihnachtsmarkt in Chemnitz nach den Gewaltvorfällen im Sommer 2018 (»Morgens halb zehn in Deutschland«, 02.09.2016; »Innere Einkehr auf Sächsisch «, 05.12.2018). In ersten Artikel wird zwar viel über Görlitz, aber nicht mit den Görlitzern gesprochen. Der Chemnitzer Artikel schildert zunächst von Uslars eigene Eindrücke, bevor er später Chemnitzer interviewt. Etwas kurios erscheint vielleicht die Überschrift als Essenz der Beobachtungen eines südafrikanischen Fotografen, der zum ersten Mal den Osten bereist, in »Land ohne Lächeln« (20.09.2017).

Die Abwertung des Ostens erfolgt im Untersuchungszeitraum zumeist indirekt über Themenkarrieren (Rechtsextremismus, AfD), die fortgesetzte Herabstufung des staatlich-institutionellen DDR-Erbes mit gleichzeitiger Anerkennung individueller Lebensleistungen und der Darstellung des Ostens als »fremdes Anderes«. Diese sind Fortführungen von etablierten Tropen mit Entstehungshintergrund im Kalten Krieg, als Wettbewerb der Selbstverständnisse darum, welches das »bessere und demokratischere Deutschland«sei.

\subsection{Diskursformation 2: Diskurse von Gleichheit}

Ein integrativer Diskurs würde theoretisch die (historisch bedingten) Unterschiede von Ost und West anerkennen und beide als gleichwertig einstufen. Dies ist im Untersuchungszeitraum gelegentlich zu beobachten. »Normalisierte Unterschiede « werden etabliert bei Frauen in Führungspositionen (so gibt es im Osten mehr »Frauen im Fußball«, 14.09.2017), bei den anderen Wünschen eines ostdeutschen Publikums der Öffentlich-Rechtlichen (»Das gestörte Programm«, 07.12.2017) und bei der Analyse zum Rechtsstatus des Schwangerschaftsabbruchs (die DDR war liberaler: »Der West-Paragraf«, 14.02.2019). Diese Diskursart erlaubt es, faktische Unterschiede zwischen Ost und West wahrzunehmen und bereitet den Boden für eine integrative Berichterstattung. 


\subsection{Diskursformation 3: Emanzipierende Diskurse}

Diese Diskurse finden sich überwiegend in Zeit im Osten, die in ihrer gedruckten Form vorrangig auf eine ostdeutsche Leser*innenschaft ausgerichtet ist. Hier wird der Osten als positiv und als Vorbild dargestellt. Beispiele dafür stammen einerseits aus einer positiven Bewertung von DDR-Gegebenheiten, andererseits aus der Gegenwart. So heißt es »Ja zur Platte« (05.11.2015) in einem Artikel über Beschränkungen in Wohnstandards, und es wird darauf verwiesen, dass die DDR-Bevölkerung weniger an Allergien litt (»Gab es vor der Wende in der DDR weniger Allergien als im Westen?«, 19.04.2017).

Für die Gegenwart dominieren positive Städtebeschreibungen, das Ostprodukt Nudossi als ökologisch vorbildlicher Brotaufstrich (»Die Zukunft aufs Brot«, 07.09.2017), RB Leipzig als erste ostdeutsche Mannschaft in der Champions League (»Auftritt der Diebe«, 08.06.2017) oder der Aufstieg von Ostdeutschen in westdeutschen Institutionen (so bei den Grünen: »Out of Guben«, 03.02.2017; oder als Vorstand der Deutschen Börse: »Immer schön flexibel«, 09.08.2018). Noch deutlicher wird dies in »Ostfrauen« (07.03.2019), des Reviews einer MDR-Serie, »die endlich zeigt, wie sehr sie dieses Land verändert haben«.

In das Bild des Ostens als positiv und »empowered« passt vor allem auch der Beitrag des Philosophen Peter Neumann (»Bitter enttäuschte Hoffnungen, nagelneue Utopien«, 17.04.2019). Anlässlich des bevorstehenden 30. Jahrestags des Mauerfalls schlug er vor, mit der Erinnerung der DDR auf eine vierte Art umzugehen - der »ostdeutsche Umbruch als europäische Utopie«. In diesem Sinne wird der Osten ein Experimentierfeld für zukünftige Gesellschaftsmodelle, ein Labor für kreative Problemlösungen, ein Ort für Innovation. Die doppelte deutsch-deutsche Erfahrung ist dabei nichts, was behindert, sondern eine wirkmächtige Ressource.

\subsection{Diskursformation 4: Artikulation von Benachteiligungen des Ostens}

Die realen Ungleichheiten zwischen Ost und West in nahezu allen Gesellschaftsbereichen finden sich nicht unbedingt im medialen Diskurs wieder. Zumindest ist die abwertende Bezeichnung »Jammerossi« (siehe Wedl 2009) aus der Zeit komplett verschwunden. 
Nach den politischen Wahlerfolgen der AfD im Osten wurde auch die Artikulation von Ungleichheit lauter. Deutlich trat dies in der Debatte um eine »Ost-Quote (2017) und gleich zweifach 2019 hervor - einerseits beim Vergleich von Ostdeutschen mit Migrant*innen und bei Vorwürfen, dass der Osten kolonialisiert wurde (»Ostquote: Verhilft sie Ostdeutschen zu mehr Chancengleichheit?«, 21.03.2019; »Ostdeutschland: Wer das Sagen hat «, 11.04.2019), andererseits bei datenjournalistischen Darstellungen zu Migrationsbewegungen (Abwanderungen) Ostdeutscher, deren Ursachen und potenziellen Rückkehrtrends (Zeitim-Osten-Special, 02.05.2019).

Gleichzeitig setzen sich Ostdeutsche mit kontinuierlicher Diskriminierung reflektiv auseinander, beispielsweise bei der Debatte um Vornamen (»Vorurteile bei Vornamen«, 03.10.2018), Doppelnamen (»Doppeldeutschland«, 07.03.2019) oder die Scham aus dem jahrzehntelangen Verstecken der Ost-Identität aus Angst vor Repressalien (»Ich bin damals abgerutscht«, 31.01.2019). Diese Momente markieren die ostdeutsche Zugehörigkeit als Thema bei einigen der Autor*innen.

Der Wandel im Diskurs ist hier bemerkenswert. Während diese Art von offenen Bekundungen und Stellungnahmen zum Einfordern von »mehr Gleichheit« 2015 nicht existierte, stellen sie inzwischen einen relevanten Teil der Artikulationen über ostdeutsche Erwartungshaltungen dar. Spätestens hier wird deutlich, dass sich Ostdeutsche nicht mehr schamvoll verstecken oder zurückziehen, sondern spürbar an Stimme gewonnen haben.

\subsection{Diskursformation 5: »Ostdeutsch sein«- Wer verhandelt die Identität?}

Dieser letzte Diskurs ist ebenso spannend wie neu. »Der Osten ist nicht totzukriegen«, schreibt der Zeit-im-Osten-Gründer Schwarz (2019b, 14) und meint damit nicht die »Alten« mit ihrer Sorge, für die eigene Biographie Spott zu ernten, sondern die Jungen, die ihre Ost-Identität teils spät für sich entdecken. Und genau dieser Punkt ist ungewöhnlich für den sonst distanziert-faktizierenden Journalismus - hier fallen plötzlich gleich mehrfach die persönliche Biographie der Zeit-Autor*innen und die journalistische Story zusammen. Es ist gerade die bewusst ausgedrückte Subjektivität, die einen Einblick in die Normalität des ostdeutschen Lebens ermöglicht - Alltagsgeschichten, die überall im Osten passieren könnten. 
Beispiele für diese autobiographischen Geschichten des Entdeckens der eigenen ostdeutschen Identität finden sich bei Caterina Lobenstein (»Deutsche Wiedervereinigung: Was blüht denn da?«, 22.06.2017), Valerie Schönian (»Heimat Ostdeutschland: Jahrgang 1990«, 14.12.2017), Martin Machowecz (»Auf der Tanne«, 14.12.2017), Maike Nedo (»Ich hab mich getarnt«, 20.12.2017; »Was wurde aus Rocco«, 15.11.2018), Sophie Herwig (»Immer auf Safari«, 28.12.2017) und - als Antwort einer anderen Ost-Generation darauf - Christoph Dieckmann (»Ostdeutschland: Jahrgang 1956«, 28.12.2017). Diese Selbstzeugnisse und offenen Beobachtungen beziehungsweise Reflektionen können Verarbeitungsstrategien von Identitätskonflikten darstellen. Doch dabei bleibt zu bedenken, dass diese generationsspezifisch sind und nicht ältere ostdeutsche Generationen repräsentieren.

Bedauerlich ist, dass ab Oktober 2017 (nach der Bundestagswahl) viele dieser so wichtigen Diskurse um ostdeutsche Identität lediglich in Zeit im Osten erscheinen, nicht jedoch in der überregionalen Printausgabe. Damit stehen Debatten um das ostdeutsche Selbstverständnis nur begrenzt einer gesamtdeutschen Leser*innenschaft zur Verfügung.

Der übrige Ost-Identitätsdiskurs bewegt sich fort von reduzierenden AfD-Assoziationen hin zum Nachdenken, was der Osten in einem gemeinsamen Deutschland sein kann und sollte. Es geht aber auch darum, wie die Wende verlaufen ist und wie wenig Westdeutsche teilweise den Osten bewusst und mit Interesse wahrnehmen (»Manche kennen den Osten der USA besser als den Osten Deutschlands«, 04.04.2019).

Diskussionen um potenziellen Kolonialismus, Parallelen zu Migrant*innen und das dynamische Aufbrechen des Ostens (»Das musste mal gesagt werden«, 14.02.2019) mündeten zwei Jahre später in der Frage, »wie viel Identitätspolitik [...] die Debatte über Ostdeutschland [verträgt] « (»Identitätspolitik. Wer wir sind. Sind wir wer?«, 14.03.2021). Die Debatte um ostdeutsche Identität ist damit nicht mehr auf den Osten beschränkt, sondern hat ein gesamtdeutsches Medium erreicht.

\section{$7 \quad$ Fazit}

Der US-amerikanische Journalismusforscher Michael Schudson (2011) schrieb, dass unabhängiger Journalismus die Grundbedingung jeder Demokratie sei. Im Vergleich zu anderen Transformationen im postsozialistischen Europa hatte der Zusammenbruch der DDR die Besonder- 
heit, dass bereits kurz nach dem Mauerfall die Presse - und später auch die Rundfunklandschaft - unter westdeutschen Eliten aufgeteilt war. Damit entstand eine Ausnahmesituation: Ostdeutsche besaßen seit 1990 nur wenig Chancen auf das ungehinderte Ausdrücken - und Verarbeiten - der eigenen Identität und der erlebten Transformationsprozesse.

Diese notwendige Arbeit leisten nun ausgerechnet die Nachgeborenen - junge ostdeutsche Journalist*innen, welche die DDR selbst nur noch vage oder gar nicht mehr miterlebt haben. Sie bringen Glaubwürdigkeit, Selbstbewusstsein und Transparenz mit, um als Mediator*innen und verspätete Integrationshelfer*innen der deutschen Einheit zu wirken, und zeigen auf, wie differenziert und innovativ der Osten sein kann. Das Negativbild steht nicht mehr allein im Vordergrund.

Unbefangen und sprachgewaltig tritt diese jüngere Generation auf. Sie kennt die DDR nur aus Geschichten, aber sie hört den jüngeren und älteren Generationen im Osten zu. Es steht zur Vermutung, dass diese Generation an Ost-Journalist*innen durch ihre geschichtliche »Unschuld « in ihren Positionen weniger angreifbar ist. Sie ringen durch das Bewusstsein der biographischen Andersartigkeit um eine Neudefinition ihrer eigenen Identität im deutsch-deutschen Gesamtgefüge. Deswegen werden Diskurse zur Emanzipation des Ostens verstärkt geführt, während jedoch elitennahe Diskurse bezüglich des negativen Erbes des DDR-Systems relativ unangetastet bleiben.

Mit dem Mauerfall 1989 wurden DDR-bezogene Identitätskonstruktionen auf dem metaphorischen »Müllhaufen der Geschichte« entsorgt - bis zum Aufstieg der AfD, die einen gesamtdeutschen Diskurs dazu animierte, doch etwas genauer auf den Osten zu schauen. Zeit im Osten ist ein Produkt eben jenes Prozesses. Der Beitrag hat gezeigt, dass es mehr als 25 Jahre gebraucht hat, bis sich die früher dokumentierten medialen Diskurse in der Öffentlichkeit verändern sollten. Diese Transformation ist gegenwärtig in vollem Gange - durch praktizierte Diversität in einer Redaktion, dem Büro von Zeit im Osten.

\section{Literatur}

Ahbe, Thomas. 2001. »Ostalgie« als Laienpraxis in Ostdeutschland. Ursachen, psychische und politische Dimensionen. In: Die DDR in Deutschland. Ein Rückblick auf 50 Jahre, herausgegeben von Heiner Timmermann, 781-802. Berlin: Duncker \& Humblot. 
Ahbe, Thomas. 2009. Die Ost-Diskurse als Strukturen der Nobilitierung und Marginalisierung von Wissen. Eine Diskursanalyse zur Konstruktion der Ostdeutschen in den westdeutschen Medien-Diskursen 1989/90 und 1995. In: Die Ostdeutschen in den Medien. Das Bild von den Anderen nach 1990, herausgegeben von Thomas Ahbe, Rainer Gries and Wolfgang Schmale, 59-112. Leipzig: Leipziger Universitätsverlag.

Ahbe, Thomas, und Rainer Gries. 2006. Die Generation der DDR und Ostdeutschlands. Ein Überblick. Berliner Debatte Initial 17 (4): 90-109.

Ahbe, Thomas, Rainer Gries, und Wolfgang Schmale. 2009. Die Ostdeutschen in den Medien. Das Bild von den Anderen nach 1990. Leipzig: Leipziger Universitätsverlag.

Belke, Julia. 2009. Das Bild der Ostdeutschen im öffentlich-rechtlichen Fernsehen. Eine Diskursanalyse des ARD-Politmagazins Kontraste in der Zeit von 19872005. In: Die Ostdeutschen in den Medien. Das Bild von den Anderen nach 1990, herausgegeben von Thomas Ahbe, Rainer Gries und Wolfgang Schmale, 135180. Leipzig: Leipziger Universitätsverlag.

Bennett, W. Lance. 2011. News: The politics of illusion. New York: Longman.

Bird, S. Elizabeth, und Robert W. Dardenne. 1988. Myth, Chronicle, and Story. Exploring the Narrative Qualities of News. In: Media, Myths, and Narratives. Television and the Press, herausgegeben von James W. Carey, 67-86. Newbury Park u. a.: Sage.

Carvalho, Anabela. 2008. Media(ted) Discourse and Society. Journalism Studies 9 (2): 161-177. DOI: 10.1080/14616700701848162.

Decker, Markus. 2017. bpb-Chef über westdeutsche Dominanz: „Es fehlen Übersetzer kultureller Differenzen«. Interview mit Thomas Krüger. Berliner Zeitung vom 31. Oktober, https://www.berliner-zeitung.de/politik-gesellschaft/bpb-chefueber-westdeutsche-dominanz-es-fehlen-uebersetzer-kultureller-differenzenli.12448. Zugegriffen: 25. März 2021.

Ergin, Yasmine. 2018. Sind Ostdeutsche auch Migranten? NDR Online vom 11. Juni, https://www.ndr.de/kultur/Sind-Ostdeutsche-auch-Migranten,migranten150.html. Zugegriffen: 1. Oktober 2018.

Fairclough, Norman. 1989. Language and Power. London: Longman.

Foroutan, Naika, Frank Kalter, Coşkun Canan, und Mara Simon. 2019. Ost-Migrantische Analogien I. Konkurrenz um Anerkennung. Stereotype, Abwertungsgefühle und Aufstiegskonflikte. Berlin: Deutsches Zentrum für Integrations- und Migrationsforschung (DeZIM).

Freunde der Zeit. 2019. Hinter der Geschichte: Der andere Blick auf Ostdeutschland. https://verlag.zeit.de/freunde/rueckblick/hinter-der-geschichte/10jahre-zeit-im-osten/. Zugegriffen: 15. März 2021.

Früh, Werner, Uwe Hasebrink, Friedrich Krotz, Christoph Kuhlmann, und HansJörg Stiehler. 1999. Ostdeutschland im Fernsehen. Erfurt: Thüringer Landesmedienanstalt.

Früh, Werner, Hans-Jörg Stiehler, Hannah Früh, und Claudia Böttcher. 2011. Mediale Vereinigungsbilanzen. Ost- und Westdeutschland im Fernsehen: Event- und Alltagsberichterstattung. Berlin: Vistas. 
Göring-Eckardt, Katrin. 2018. Bleibende Unterschiede. Deutschlands Osten ist anders. Wirtschaftswoche vom 22. September, https://www.wiwo.de/my/politik/ deutschland/bleibende-unterschiede-deutschlands-osten-ist-anders/23089018.html?ticket=ST-653181-Vt71Q51fgjXvQFB9Gu1h-ap2. Zugegriffen: 25. März 2021.

Halbwachs, Maurice. 1992. On Collective Memory. Chicago, London: University of Chicago Press.

Hobsbawm, Eric, und Terence Ranger. 1983. The Invention of Tradition. Cambridge: Cambridge University Press.

Hogwood, Patricia. 2013. Selective Memory. Channeling the Past in Post-GDR Society. In: Remembering and Rethinking the GDR. Multiple Perspectives and Plural Authenticities, herausgegeben von Anna Saunders and Debbie Pinfold, 34-48. Houndmills, Basingstoke, New York: Palgrave Macmillan.

Hörschelmann, Kathrin. 2001. Breaking ground-marginality and resistance in (post) unification Germany. Political Geography 20 (8): 981-1004. DOI: https:// doi.org/10.1016/S0962-6298(01)00041-5.

Köhler, Sebastian. 2009. Die Nachrichtenerzähler. Zu Theorie und Praxis nachhaltiger Narrativität im TV-Journalismus. Baden-Baden: Nomos / Edition Reinhard Fischer.

Kollmorgen, Raj, Frank-Thomas Koch, und Hans-Liudger Dienel. 2011. Diskurse der deutschen Einheit. Kritik und Alternativen. Wiesbaden: VS Verlag für Sozialwissenschaften.

Kolmer, Christian. 2009. Nachrichten aus einer Krisenregion. Das Bild Ostdeutschlands und der DDR in den Medien 1994-2007. In: Die Ostdeutschen in den Medien. Das Bild von den Anderen nach 1990, herausgegeben von Thomas Ahbe, Rainer Gries und Wolfgang Schmale, 181-214. Leipzig: Leipziger Universitätsverlag.

Köpping, Petra. 2018. Integriert doch erstmal uns! Eine Streitschrift für den Osten. Berlin: Christoph Links Verlag.

Kubiak, Daniel, und Martin Weinel. 2016. DDR-Generationen revisited - Gibt es einen Generationszusammenhang der »Wendekinder«? In: Die Generation der Wendekinder. Elaboration eines Forschungsfeldes, herausgegeben von Adriana Lettrari, Christian Nestler and Nadja Troi-Boeck, 107-129. Springer VS.

Langer, Susanne K. 1965. Philosophie auf neuem Wege. Das Sympol im Denken, im Ritus und der Kunst. Frankfurt am Main: S. Fischer.

Liebscher, Doris. 2019. »Wir sind ein Volk«? Warum wir Rechtsschutz gegen die Diskriminierung als »Ossi« brauchen. Humboldt Law Clinic, Grundundmenschenrechtsblog vom 17. Oktober, http://grundundmenschenrechtsblog.de/ wir-sind-ein-volk-warum-wir-rechtsschutz-gegen-die-diskriminierung-als-ossibrauchen/. Zugegriffen: 18. Januar 2021.

Machin, David, und Andrea Mayr. 2012. How To Do Critical Discourse Analysis. A Multimodal Introduction. Los Angeles, London u. a.: Sage.

Mannheim, Karl. 1980. Strukturen des Denkens. Frankfurt am Main: Suhrkamp.

Matejskova, Tatiana. 2013. The Unbearable Closeness of The East: Embodied MicroEconomies of Difference, Belonging, and Intersecting Marginalities in Post-Socialist Berlin. Urban Geography 34 (1): 30-52. DOI: 10.1080/02723638.2013.778630. 
MDR. 2018. Analyse zur TV-Doku »Wer braucht den Osten?« Ostdeutschland in der Presse. Mitteldeutscher Rundfunk vom 9. Juni, https://www.mdr.de/zeitreise/ wer-braucht-den-osten-datenanalyse-der-osten-in-den-medien-100.html. Zugegriffen: 17. März 2021.

Meier, Klaus. 2018. Journalistik. 4. Auflage. Konstanz: UTB.

Meyen, Michael. 2013. »Wir haben freier gelebt«. Die DDR im kollektiven Gedächtnis der Deutschen. Bielefeld: Transcript.

Mükke, Lutz. 2021. 30 Jahre staatliche Einheit - 30 Jahre mediale Spaltung. Schreiben Medien die Teilung Deutschlands fest? OBS Arbeitspapier. Frankfurt am Main: Otto-Brenner-Stiftung, https://www.otto-brenner-stiftung.de/fileadmin/user_ data/stiftung/02_Wissenschaftsportal/03_Publikationen/AP45_Mediale_Spaltung.pdf. Zugegriffen: 26. März 2021.

Neues Deutschland. 2001. Ostjugend: In: DDR war es nicht schlechter. Neues Deutschland vom 8. Februar, https://www.neues-deutschland.de/artikel/899447.ostjugend-in-ddr-war-es-nicht-schlechter.html. Zugegriffen: 26. März 2021.

Oberender, Thomas. 2017. Deutsche Einheit: Die Mauer ist nicht gefallen. Die Zeit vom 3. Oktober, https://www.zeit.de/2017/40/ddr-mauerfall-kinderbetreuung-west-ost-deutschland. Zugegriffen: 14. Februar 2021

Ondreka, Lukas. 2015. Joachim Gauck, die Ossis und Dunkeldeutschland. Süddeutsche Zeitung vom 26. August, https://www.sueddeutsche.de/politik/bundespraesident-joachim-gauck-die-ossis-und-dunkeldeutschland-1.2622780. Zugegriffen: 14. Februar 2021.

Presse- und Informationsamt der Bundesregierung. 2020. 30 Jahre Deutsche Einheit. Schwarzrotgold. Das Magazin der Bundesregierung, Heft 2, https://www. bundesregierung.de/resource/blob/975232/1779314/e933561f3e0a91ce366a cccc392632aa/download-pdf-data.pdf?download=1. Zugegriffen: 14. Februar 2021

Richter, Frank. 2018. Hört endlich zu! Weil Demokratie Auseinandersetzung bedeutet. Berlin: Ullstein.

Roth, Kersten Sven. 2008. Der Westen als »Normal Null«. Zur Diskurssemantik von »ostdeutsch« und »westdeutsch«. In: Diskursmauern. Aktuelle Aspekte der sprachlichen Verhältnisse zwischen Ost und West, herausgegeben von Kersten Sven Roth and Markus Wienen, 69-89. Bremen: Dr. Ute Hempen Verlag.

Ruhrmann, Georg. 2016. Das Bild Ostdeutschlands in den Medien. Von der Unterrepräsentation zur »Stigmatisierung«? In: Empirische Medienökonomie. Reflexionen der Arbeiten von Wolfgang Seufert, herausgegeben von Jörg Müller-Lietzkow and Felix Sattelberger, 138-160. Baden-Baden: Nomos.

Sabrow, Martin. 2009. Vorwort. Die DDR erinnern. In: Erinnerungsorte der DDR, herausgegeben von Martin Sabrow, 11-27. München: C. H. Beck.

Schudson, Michael. 2011. The Sociology of News. New York, London: W. W. Norton \& Company.

Schwarz, Patrik. 2019a. Guter Osten, böser Osten. Bonn: Bundeszentrale für politische Bildung.

Schwarz, Patrik. 2019b. Nicht wegen der Alten, sondern wegen der Jungen gibt es 
den Osten noch. In: Guter Osten, böser Osten, herausgegeben von Patrik Schwarz, 14-15. Bonn: Bundeszentrale für politische Bildung.

Tenenboim-Weinblatt, Keren. 2011. Journalism as Agent of Prospective Memory. In: On Media Memory. Collective Memory in a New Media Age, herausgegeben von Motti Neiger, Oren Meyers und Eyal Zandberg, 213-225. Basingstoke: Palgrave Macmillan.

Tröger, Mandy. 2019. Pressefrühling und Profit: Wie westdeutsche Verlage 1989/1990 den Osten eroberten. Köln: Herbert von Halem.

Wedl, Juliette. 2009. Ein Ossi ist ein Ossi ist ein Ossi ... Regeln der medialen Berichterstattung über »Ossis« und »Wessis« in der Wochenzeitung Die Zeit seit Mitte der 1990er Jahre. In: Die Ostdeutschen in dem Medien. Das Bild von den Anderen nach 1990, herausgegeben von Thomas Ahbe, Rainer Gries und Wolfgang Schmale, 112-134. Leipzig: Leipziger Universitätsverlag.

Wippermann, Wolfgang. 2009. Dämonisierung durch Vergleich: DDR und Drittes Reich. Berlin: Rotbuch.

\section{Open Access}

Dieser Beitrag erscheint unter der Creative-Commons-Lizenz CC BY-ND 3.0 DE: https://creativecommons.org/licenses/by-nd/3.0/de/. 